\title{
Quantum Block and Convolutional Codes from Self-orthogonal Product Codes
}

\author{
Markus Grassl \\ Institut für Algorithmen und Kognitive Systeme \\ Arbeitsgruppe Quantum Computing \\ Fakultät für Informatik, Universität Karlsruhe (TH) \\ Am Fasanengarten 5, 76128 Karlsruhe, Germany \\ Email: grass1@ira.uka.de
}

\author{
Martin Rötteler \\ NEC Labs America, Inc. \\ 4 Independence Way \\ Princeton, NJ 08540, USA \\ Email: mroetteler@nec-labs.com
}

\begin{abstract}
We present a construction of self-orthogonal codes using product codes. From the resulting codes, one can construct both block quantum error-correcting codes and quantum convolutional codes. We show that from the examples of convolutional codes found, we can derive ordinary quantum error-correcting codes using tail-biting with parameters $\llbracket 42 N, 24 N, 3 \rrbracket_{2}$. While it is known that the product construction cannot improve the rate in the classical case, we show that this can happen for quantum codes: we show that a code $\llbracket 15,7,3 \rrbracket_{2}$ is obtained by the product of a code $\llbracket 5,1,3 \rrbracket_{2}$ with a suitable code.
\end{abstract}

\section{INTRODUCTION}

Quantum convolutional codes are motivated by their classical counterparts [3]. As in the classical case the idea is to allow for the protection of arbitrary long streams of information in such a way that as many errors as possible can be corrected. To achieve this the information is "smeared out" to the output stream by adding a certain amount of redundancy, but at the same time meeting the requirement to be local, i.e., encoding/decoding can be done by a processes which needs only a constant amount of memory. In [13] the basic theory of quantum convolutional codes has been developed. There it has been shown that, similar to the classical codes, quantum convolutional codes can be decoded by a maximum likelihood error estimation algorithm which has linear complexity. However, the authors only gave an example of one (rate 1/5) quantum convolutional code. This research was motivated by the question to find new examples of quantum convolutional codes. The construction presented in this paper resorts on the idea of product codes. An extra requirement imposed by the applicability to quantum codes is that the dual distance has to be high. The main source of the examples presented at the end of the paper are two-dimensional cyclic codes (sometimes also called "bicyclic codes"). We apply this to the situation where the code is a product code of two Reed-Solomon codes.

\section{Self-orthogonal Product Codes}

\section{A. Quantum error-correcting codes from classical codes}

Most of the constructions for quantum error-correcting codes (QECCs) for a quantum system of dimension $q$ (qudits), where $q=p^{\ell}$ is a prime power, are based on classical errorcorrecting codes over $G F(q)$ or $G F\left(q^{2}\right)$. The so-called CSS codes (see [5], [14]) are based on linear codes $C_{1}$ and $C_{2}$ over $G F(q)$ with $C_{2}^{\perp} \subseteq C_{1}$. Here $C_{2}^{\perp}$ is the dual code of $C_{2}$ with respect to the Euclidean inner product. In particular, if $C=C_{1}=C_{2}$ this implies that $C^{\perp}$ is a weakly self-dual code. The construction can be summarized as follows:

Lemma 1: Let $C=[n, k, d]_{q}$ be a weakly self-dual linear code, i. e., $C \subseteq C^{\perp}=\left[n, n-k, d^{\perp}\right]_{q}$. Then a quantum errorcorrecting code encoding $n-2 k$ qudits using $n$ qudits, denoted by $\mathcal{C}=\llbracket n, n-2 k, d_{q} \geq d^{\perp} \rrbracket_{q}$ exists.

Another class of quantum codes can be obtained from codes over $G F\left(q^{2}\right)$ which are self-orthogonal with respect to the Hermitian inner product, denoted by $C \subseteq C^{*}$. Both cases can be generalized to a construction of QECCs based on additive codes over $G F\left(q^{2}\right)$ which are self-orthogonal with respect to the symplectic (trace) inner product, i. e. $C \subseteq C^{\star}[1]$.

B. Inner products on vector spaces over $G F(q)$ and $G F\left(q^{2}\right)$

In this paper, we will use three different inner products on vector spaces over $G F(q)$ and $G F\left(q^{2}\right)$ which are defined as follows:

Euclidean:

$$
\boldsymbol{v} \cdot \boldsymbol{w}:=\sum_{i=1}^{n} v_{i} w_{i} \quad \text { for } \boldsymbol{v}, \boldsymbol{w} \in G F(q)^{n}
$$

Hermitian:

$$
\boldsymbol{v} * \boldsymbol{w}:=\sum_{i=1}^{n} v_{i} w_{i}^{q} \quad \text { for } \boldsymbol{v}, \boldsymbol{w} \in G F\left(q^{2}\right)^{n}
$$

symplectic:

$$
\boldsymbol{v} \star \boldsymbol{w}:=\sum_{i=1}^{n} \operatorname{tr}\left(v_{i} w_{i}^{q}\right) \quad \text { for } \boldsymbol{v}, \boldsymbol{w} \in G F\left(q^{2}\right)^{n},
$$

where $\operatorname{tr}(x)$ denotes the trace of $G F\left(q^{2}\right)$ over its prime field $G F(p)$. Both the Euclidean and the Hermitian inner product are bilinear over $G F(q)$ respectively $G F\left(q^{2}\right)$, but the symplectic inner product is only $G F(p)$-bilinear because of the trace map. For codes which are linear over $G F(q)$, linear over $G F\left(p^{2}\right)$, or additive (i.e. $G F(p)$-linear), one can define a dual code with respect to the inner products (1), (2), or (3), respectively. The three cases are summarized in Table \.

Next, we consider inner products on tensor products of vector spaces. 
TABLE I

NOTATION USED FOR THE THREE DIFFERENT INNER PRODUCTS AND THE CORRESPONDING DUAL CODES.

\begin{tabular}{l|c|c|c} 
& dual code & inner product & linear over \\
\hline Euclidean & $C^{\perp}$ & $\boldsymbol{v} \cdot \boldsymbol{w}$ & $G F(q)$ \\
Hermitian & $C^{*}$ & $\boldsymbol{v} * \boldsymbol{w}$ & $G F\left(q^{2}\right)$ \\
symplectic & $C^{\star}$ & $\boldsymbol{v} \star \boldsymbol{w}$ & $G F(p)$
\end{tabular}

Lemma 2: For all $\boldsymbol{v}, \boldsymbol{v}^{\prime} \in G F(q)^{n}$ and $\boldsymbol{w}, \boldsymbol{w}^{\prime} \in G F(q)^{m}$, we have

$$
(\boldsymbol{v} \otimes \boldsymbol{w}) \cdot\left(\boldsymbol{v}^{\prime} \otimes \boldsymbol{w}^{\prime}\right)=\left(\boldsymbol{v} \cdot \boldsymbol{v}^{\prime}\right)\left(\boldsymbol{w} \cdot \boldsymbol{w}^{\prime}\right),
$$

i. e., the Euclidean inner product is compatible with the tensor product of vector spaces over $G F(q)$. Furthermore, for all $\boldsymbol{v}, \boldsymbol{v}^{\prime} \in G F\left(q^{2}\right)^{n}$ and $\boldsymbol{w}, \boldsymbol{w}^{\prime} \in G F\left(q^{2}\right)^{m}$, we have

$$
(\boldsymbol{v} \otimes \boldsymbol{w}) *\left(\boldsymbol{v}^{\prime} \otimes \boldsymbol{w}^{\prime}\right)=\left(\boldsymbol{v} * \boldsymbol{v}^{\prime}\right)\left(\boldsymbol{w} * \boldsymbol{w}^{\prime}\right),
$$

i. e., the Hermitian inner product is compatible with the tensor product of vector spaces over $G F\left(q^{2}\right)$.

Proof: The tensor product of two vectors is given by $(\boldsymbol{v} \otimes \boldsymbol{w})=\left(v_{i} w_{j}\right)_{i, j}$. Then for the Euclidean inner product we get

$$
\begin{aligned}
(\boldsymbol{v} & \otimes \boldsymbol{w}) \cdot\left(\boldsymbol{v}^{\prime} \otimes \boldsymbol{w}^{\prime}\right) \\
& =\sum_{i=1}^{n} \sum_{j=1}^{m} v_{i} w_{j} v_{i}^{\prime} w_{j}^{\prime}=\left(\sum_{i=1}^{n} v_{i} v_{i}^{\prime}\right)\left(\sum_{j=1}^{m} w_{j} w_{j}^{\prime}\right) \\
& =\left(\boldsymbol{v} \cdot \boldsymbol{v}^{\prime}\right)\left(\boldsymbol{w} \cdot \boldsymbol{w}^{\prime}\right) .
\end{aligned}
$$

Similarly, for the Hermitian inner product we get

$$
\begin{aligned}
(\boldsymbol{v} & \otimes \boldsymbol{w}) *\left(\boldsymbol{v}^{\prime} \otimes \boldsymbol{w}^{\prime}\right) \\
& =\sum_{i=1}^{n} \sum_{j=1}^{m} v_{i} w_{j}\left(v_{i}^{\prime} w_{j}^{\prime}\right)^{q}=\left(\sum_{i=1}^{n} v_{i} v_{i}^{\prime q}\right)\left(\sum_{j=1}^{m} w_{j} w_{j}^{\prime q}\right) \\
& =\left(\boldsymbol{v} * \boldsymbol{v}^{\prime}\right)\left(\boldsymbol{w} * \boldsymbol{w}^{\prime}\right) .
\end{aligned}
$$

For the symplectic inner product, the situation is a bit more complicated as it is only $G F(p)$-linear. Considering $G F(q)^{m}$ only as vector space over $G F(p)$, we may define the $G F(p)$ tensor product of $V_{1}=G F(p)^{n}$ and $V_{2}=G F(q)^{m}$, denoted by $V_{1} \otimes_{p} V_{2}$.

Lemma 3: For all $\boldsymbol{v}, \boldsymbol{v}^{\prime} \in G F(p)^{n}$ and $\boldsymbol{w}, \boldsymbol{w}^{\prime} \in G F(q)^{m}$, we have $\left(\boldsymbol{v} \otimes_{p} \boldsymbol{w}\right) \star\left(\boldsymbol{v}^{\prime} \otimes_{p} \boldsymbol{w}^{\prime}\right)=\left(\boldsymbol{v} \cdot \boldsymbol{v}^{\prime}\right)\left(\boldsymbol{w} \star \boldsymbol{w}^{\prime}\right)$, i.e., the symplectic inner product on the $G F(p)$ tensor product space is the product of the Euclidean inner product on the first space and the symplectic inner product on the second.

Proof: Similar to the proof of Lemma 2, we compute

$$
\begin{aligned}
(\boldsymbol{v} \otimes \boldsymbol{w}) \star\left(\boldsymbol{v}^{\prime} \otimes \boldsymbol{w}^{\prime}\right) & =\sum_{i=1}^{n} \sum_{j=1}^{m} \operatorname{tr}\left(v_{i} w_{j}\left(v_{i}^{\prime} w_{j}^{\prime}\right)^{q}\right) \\
& =\operatorname{tr}\left(\left(\sum_{i=1}^{n} v_{i} v_{i}^{\prime q}\right)\left(\sum_{j=1}^{m} w_{j} w_{j}^{\prime q}\right)\right) .
\end{aligned}
$$

As $\boldsymbol{v}$ and $\boldsymbol{v}^{\prime}$ are vectors over the prime field, the left factor equals their Euclidean inner product $\boldsymbol{v} \cdot \boldsymbol{v}^{\prime}$ which takes values in $G F(p)$ only. Using the $G F(p)$-linearity of the trace map, the proof is completed.

\section{Product codes}

Next we present the fundamental properties of the product of two codes which combines two codes (see e. g. [2], [11]).

Lemma 4: Let $C_{1}=\left[n_{1}, k_{1}, d_{1}\right]_{q}$ and $C_{2}=\left[n_{2}, k_{2}, d_{2}\right]_{q}$ be linear codes over $G F(q)$ with generator matrices $G^{(1)}$ and $G^{(2)}$, respectively. Then the product code $C_{\pi}:=C_{1} \otimes C_{2}$ is a linear code $C_{\pi}:=\left[n_{1} n_{2}, k_{1} k_{2}, d_{1} d_{2}\right]_{q}$ generated by the matrix $G:=G^{(1)} \otimes G^{(2)}$, where $\otimes$ denotes the Kronecker product, i.e.

$$
G:=\left(\begin{array}{cccc}
g_{11}^{(1)} G^{(2)} & g_{12}^{(1)} G^{(2)} & \ldots & g_{1, n_{1}}^{(1)} G^{(2)} \\
g_{21}^{(1)} G^{(2)} & g_{22}^{(1)} G^{(2)} & \ldots & g_{2, n_{1}}^{(1)} G^{(2)} \\
\vdots & \vdots & \ddots & \vdots \\
g_{k_{1}, 1}^{(1)} G^{(2)} & g_{k_{1}, 2}^{(1)} G^{(2)} & \ldots & g_{k_{1}, n_{1}}^{(1)} G^{(2)}
\end{array}\right)
$$

If $C_{1}=\left[n_{1}, k_{1}, d_{1}\right]_{p}$ is a linear code over the prime field $G F(p)$ and $C_{2}=\left(n_{2}, p^{k_{2}}, d_{2}\right)_{q}$ is an additive code over $G F(q)$, then $C_{\pi, p}:=C_{1} \otimes_{p} C_{2}$ is an additive code with parameters $C_{\pi, p}=\left(n_{1} n_{2}, p^{k_{1} k_{2}}, d_{1} d_{2}\right)_{q}$.

The following theorem is valid for all compatible choices of inner products on the component spaces of a tensor product space and the tensor product space itself.

Theorem 5: Let $C_{\pi}=C_{1} \otimes C_{2}$ be the product code of the codes $C_{1}=\left[n_{1}, k_{1}, d_{1}\right]$ and $C_{2}=\left[n_{2}, k_{2}, d_{2}\right]$. By $H_{1}$ and $H_{2}$ we denote generator matrices of the corresponding dual codes. Furthermore, let $A_{1}$ and $A_{2}$ be matrices of size $k_{1} \times n_{1}$ and $k_{2} \times n_{2}$, respectively, such that the row span of the matrices $H_{1}$ and $A_{1}$ is the full vector space and similar for $H_{2}$ and $A_{2}$. Then a generator matrix $H$ of the dual code of $C_{\pi}$ is given by

$$
H:=\left(\begin{array}{c}
H_{1} \otimes H_{2} \\
A_{1} \otimes H_{2} \\
H_{1} \otimes A_{2}
\end{array}\right) .
$$

Proof: Let $V_{1}$ and $V_{2}$ be the full vector spaces containing the codes $C_{1}$ and $C_{2}$. Furthermore, by $D_{1}$ and $D_{2}$ we denote the dual code of $C_{1}$ and $C_{2}$ with respect to the inner product on $V_{1}$ and $V_{2}$, respectively. Using the properties of the inner products on tensor product spaces (see Lemma2 and Lemma 3), it is obvious that the dual code $D_{\pi}$ of $C_{\pi}$ contains both $V_{1} \otimes D_{2}$ and $D_{1} \otimes V_{2}$. The intersection of these spaces is $D_{0}:=D_{1} \otimes D_{2}$, spanned by $H_{1} \otimes H_{2}$. The complement of $D_{0}$ in $V_{1} \otimes D_{2}$ is spanned by $A_{1} \otimes H_{2}$, and analogously for the complement of $D_{0}$ in $D_{1} \otimes V_{2}$. Hence $D_{\pi}$ can be decomposed as

$$
D_{\pi}=\left(D_{1} \otimes D_{2}\right) \oplus\left(\left\langle A_{1}\right\rangle \otimes D_{2}\right) \oplus\left(D_{1} \otimes\left\langle A_{2}\right\rangle\right) .
$$

Here $\langle A\rangle$ denotes the row span of the matrix $A$. Considering the dimension of the spaces, the result follows. 
Corollary 6: The minimum distance of the dual of the product code $C_{\pi}=C_{1} \otimes C_{2}$ cannot exceed the minimum of the dual distance of $C_{1}$ and the dual distance of $C_{2}$.

Proof: The dual code $D_{\pi}$ of $C_{\pi}$ contains $V_{1} \otimes D_{2}$, i.e., the product of the trivial code $\left[n_{1}, n_{1}, 1\right]$ and $D_{2}$. Hence the minimum distance of $D_{\pi}$ cannot be larger than that of $D_{2}$. The result follows by interchanging the role of $C_{1}$ and $C_{2}$.

Note that despite their poor behavior in terms of minimum distance, the dual of product codes can be used for burst error correction (see [6], [15]). For the construction of QECCs, we will make use of the following property.

Theorem 7: Let $C_{E} \subseteq C_{E}^{\perp}, C_{H} \subseteq C_{H}^{*}$, and $C_{s} \subseteq C_{s}^{\star}$ denote codes which are self-orthogonal with respect to the inner products (1), (2), or (3), respectively. Furthermore, let $C$ denote an arbitrary linear code over $G F(q)$, respectively $G F\left(q^{2}\right)$, and let $C_{p}$ be a linear code over $G F(p)$. Then

(i) $C \otimes C_{E}$ is Euclidean self-orthogonal.

(ii) $C \otimes C_{H}$ is Hermitian self-orthogonal.

(iii) $C_{p} \otimes_{p} C_{s}$ is symplectic self-orthogonal.

Proof: The result directly follows using Lemma 2 Lemma 3, and Theorem 5 .

\section{Product Codes from Cyclic Codes}

In this section we investigate the product of two cyclic codes (see [2, Chapter 10.4], [3, Chapter 10.2]).

Let $C_{1}=\left[n_{1}, k_{1}\right]$ and $C_{2}=\left[n_{2}, k_{2}\right]$ be cyclic linear codes with generator polynomials $g_{1}(X)$ and $g_{2}(Y)$. Then $C_{\pi}=C_{1} \otimes C_{2}$ is a bicyclic code generated by $g_{1}(X) g_{2}(Y)$. The codewords of $C_{\pi}$ correspond to all bivariate polynomials $c(X, Y)=i(X, Y) g_{1}(X) g_{2}(Y)$ modulo the ideal generated by $X^{n_{1}}-1$ and $Y^{n_{2}}-1$, where $i(X, Y) \in G F(q)[X, Y]$ is an arbitrary bivariate polynomial. The two-dimensional spectrum of $c(X, Y)$ is the $n_{1} \times n_{2}$ matrix $\left(\hat{c}_{i, j}\right)$ with entries

$$
\hat{c}_{i, j}:=c\left(\alpha^{i}, \beta^{j}\right) \text {, }
$$

where $\alpha$ and $\beta$ are primitive roots of unity of order $n_{1}$ and $n_{2}$, respectively. The spectrum $\hat{c}$ is zero in all vertical stripes corresponding to the roots $\alpha^{i}$ of $g_{1}(X)$ and in all horizontal stripes corresponding to the roots $\beta^{j}$ of $g_{2}(X)$ (see Fig. 1 a)). The generator polynomial $h_{1}(X)$ of the Euclidean dual $C_{1}^{\perp}$ is the reciprocal polynomial of $\left(X^{n_{1}}-1\right) / g_{1}(X)$. Hence its one-dimensional spectrum is zero at the negative of those positions where the spectrum of the code $C_{1}$ takes arbitrary values ( $c f$. Fig. 2). For the generator polynomial $h_{2}(Y)$ of $C_{2}^{\perp}$ the analogous statement is true. Therefore the Euclidean dual code $\left(C_{1} \otimes C_{2}\right)^{\perp}$ of the product code $C_{1} \otimes C_{2}$ consists of all polynomials that are multiples of $h_{1}(X)$ or $h_{2}(Y)$. Interchanging the zeros and blanks in the two-dimensional spectrum of the product code and applying the coordinate map (cf. Fig. 2) to both the rows and columns, we obtain the twodimensional spectrum of the dual code $\left(C_{1} \otimes C_{2}\right)^{\perp}$.

For the Hermitian dual code, we get analogous results. As the Hermitian inner product involves the Frobenius map $x \mapsto x^{q}$, the transformation on the coordinates now reads $i \mapsto-q i \bmod n_{j}$.

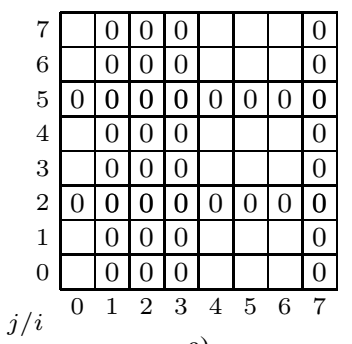

a)

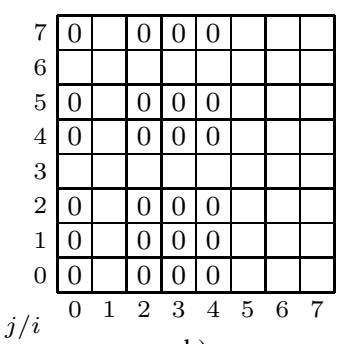

b)
Fig. 1. Two-dimensional spectrum of a) the product of two cyclic codes and b) the dual code. Blank entries may take arbitrary value.

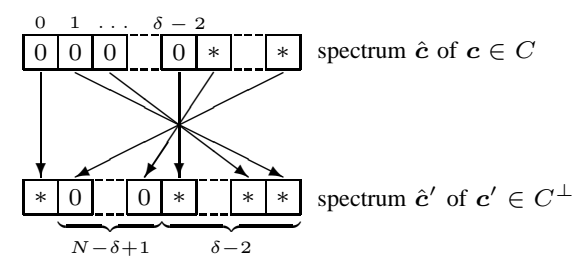

Fig. 2. Relation between the spectra of a Reed-Solomon code $C$ and its dual. Positions taking arbitrary values (marked with $*$ ) and positions being zero are interchanged using to the map $i \mapsto-i \bmod (q-1)$ [9].

For Reed-Solomon codes, the picture simplifies. The twodimensional spectrum of the product of two Reed-Solomon codes with minimum distance $\delta_{1}$ and $\delta_{2}$ corresponds to a vertical stripe of zeros of width $\delta_{1}-1$ and a horizontal stripe of height $\delta_{2}-1$. Without loss of generality, the stripes can be shifted such that the rectangle of arbitrary values is in the upper right corner (see Fig. 3 a). Then for the dual code, the spectrum is zero in a rectangle (see Fig. 3 b) whose width and height is determined by the dual distances $\left(q-\delta_{1}\right)$ and $\left(q-\delta_{1}\right)$. Using the BCH-like lower bound for bicyclic codes (see [3, p. 320]), we conclude that the minimum distance of the dual of the product code is $\min \left(q-\delta_{1}, q-\delta_{2}\right)$. In summary, we get the following theorem:

Theorem 8: The product code of two Reed-Solomon codes $C_{1}=\left[q-1, q-\delta_{1}, \delta_{1}\right]_{q}$ and $C_{2}=\left[q-1, q-\delta_{2}, \delta_{2}\right]_{q}$ over $G F(q)$ is

$$
C_{1} \otimes C_{2}=\left[(q-1)^{2},\left(q-\delta_{1}\right)\left(q-\delta_{2}\right), \delta_{1} \delta_{2}\right]_{q} .
$$

The Euclidean dual code $\left(C_{1} \otimes C_{2}\right)^{\perp}=\left[(q-1)^{2}, K^{\perp}, d^{\perp}\right]_{q}$

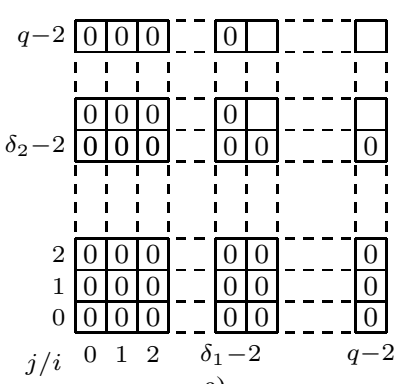

a)

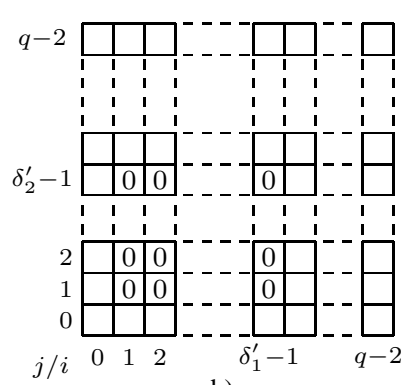

b)
Fig. 3. Two-dimensional spectrum of a) the product of two Reed-Solomon codes $C_{1}$ and $C_{2}$ with minimum distance $\delta_{1}$ and $\delta_{2}$, and b) the dual code, where $\delta_{1}^{\prime}$ and $\delta_{2}^{\prime}$ denote minimum distance of the dual codes $C_{1}^{\perp}$ and $C_{2}^{\perp}$. 
has parameters

$$
\begin{aligned}
K^{\perp} & =q\left(d_{1}+d_{2}-2\right)-d_{1} d_{2}+1 \\
d^{\perp} & =\min \left(q-\delta_{1}, q-\delta_{2}\right) .
\end{aligned}
$$

Moreover, the product code is self-orthogonal if $C_{1}$ or $C_{2}$ is self-orthogonal.

Note that the result is still true when replacing the ReedSolomon code over $G F(q)$ of length $(q-1)$ by a cyclic code $C=[n, k, d]_{q}$ with generator polynomial $g(X)=$ $\prod_{i=0}^{d-2}\left(X-\alpha^{i}\right)$ where $n$ is a divisor of $q-1$ and $\alpha$ is a primitive $n$-th root of unity.

\section{Quantum Codes From Product Codes}

\section{A. Quantum Block Codes}

In the previous section we have seen that the product of a self-orthogonal Reed-Solomon code with an arbitrary ReedSolomon codes yields a self-orthogonal product code. Using Lemma 1, we can construct quantum error-correcting codes.

Theorem 9: Let $C_{1}=\left[q-1, \mu_{1}, q-\mu_{1}\right]_{q}$ and $C_{2}=[q-$ $\left.1, \mu_{2}, q-\mu_{2}\right]_{q}$ be Reed-Solomon codes where $\mu_{1}<(q-1) / 2$. Then a quantum error-correcting code

$$
\mathcal{C}=\llbracket(q-1)^{2},(q-1)^{2}-2 \mu_{1} \mu_{2}, 1+\min \left(\mu_{1}, \mu_{2}\right) \rrbracket_{q}
$$

exists.

Proof: For $\mu_{1}<(q-1) / 2$, the code $C_{1}$ is Euclidean selforthogonal [10]. The dual distance of $C_{1}$ and $C_{2}$ is $\mu_{1}+1$ and $\mu_{2}+1$, respectively. By Theorem 8 , the product code $C_{\pi}=C_{1} \otimes C_{2}=\left[(q-1)^{2}, \mu_{1} \mu_{2},\left(q-\mu_{1}\right)\left(q-\mu_{2}\right)\right]_{q}$ is selforthogonal. Its Euclidean dual has parameters $C_{\pi}^{\perp}=[(q-$ $\left.1)^{2},(q-1)^{2}-\mu_{1} \mu_{2}, 1+\min \left(\mu_{1}, \mu_{2}\right)\right]_{q}$. Hence by Lemma 1 a QECC with the parameters given in eq. (10) exists.

Note that from $C_{1}$ and $C_{2}$ (provided $\mu_{2}<(q-1) / 2$ ), one can construct optimal QECCs with parameters $\llbracket q-1, q-2 \mu-$ $1, \mu+1 \rrbracket_{q}$ (see [10]). The product of the rates of these codes is

$$
\left(1-\frac{2 \mu_{1}}{q-1}\right)\left(1-\frac{2 \mu_{2}}{q-1}\right)=1-\frac{2\left(\mu_{1}+\mu_{2}\right)}{q-1}+\frac{4 \mu_{1} \mu_{2}}{(q-1)^{2}}
$$

The rate of the code of Theorem 9 is

$$
1-\frac{2 \mu_{1} \mu_{2}}{(q-1)^{2}} \text {. }
$$

If we choose $\mu_{1}=\mu_{2}$, we will obtain a QECC of squared length and the same minimum distance, but higher rate provided $\mu_{1}=\mu_{2}<2(q-1) / 3$.

Note that we can obtain good QECCs by this construction using other codes than Reed-Solomon codes. Let $C=$ $[5,2,4]_{4}$ be the Hermitian dual of the quaternary Hamming code. Using $C \subseteq C^{*}=[5,3,3]_{4}$, an optimal QECC $\mathcal{C}=$ $\llbracket 5,1,3 \rrbracket_{2}$ can be constructed. The code $C$ is not a ReedSolomon code, but its spectrum fulfills the conditions for Theorem 8 . Hence the product of $C$ with itself is a Hermitian self-orthogonal code $C \otimes C=[25,4,16]_{4} \subseteq(C \otimes C)^{*}=$ $[25,21,3]_{4}$. This yields a QECC $\mathcal{C}^{(2)}=\llbracket 25,17,3 \rrbracket_{2}$, whose rate is more than three times higher than that of $\mathcal{C}$.
The product code of $C$, considered as additive code, with the binary simplex code $C_{1}=[3,2,2]_{2}$ is an additive code $C_{2}:=$ $C_{1} \otimes_{p} C=\left(15,2^{8}, 8\right)_{2}$ which is contained in its symplectic dual $C^{\star}=\left(15,2^{22}, 3\right)_{2}$. Hence we obtain a QECC $\mathcal{C}_{\pi}=$ $\llbracket 15,7,3 \rrbracket_{2}$.

\section{Quantum Convolutional Codes}

Following [13], an $(n, k, m)$ quantum convolutional code can be described in terms of a semi-infinite stabilizer matrix $S$. The matrix $S$ has a block band structure where each block $M$ has size $(n-k) \times(n+m)$. All blocks are equal. In the second block, the matrix $M$ is shifted by $n$ columns, hence any two consecutive blocks overlap in $m$ positions. The general structure of the matrix is as follows:

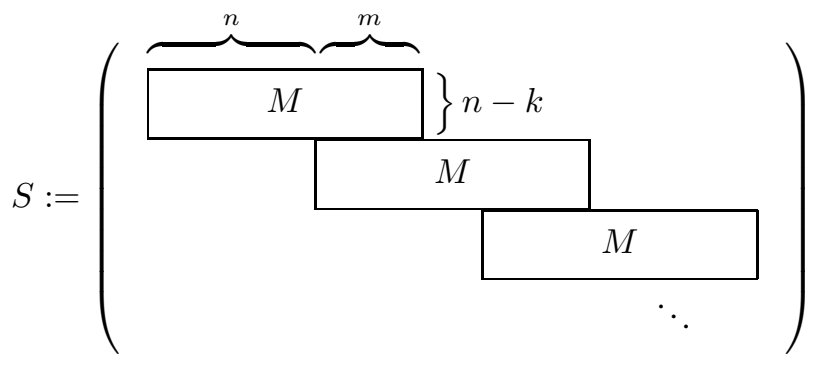

The classical convolutional code generated by $S$ must be self-orthogonal with respect to some of the inner products of Section The quantum product codes constructed in the previous section naturally lend themselves to convolutional codes because of the following observation. Let $M=G^{(1)} \otimes G^{(2)}$ be the generator matrix of $C_{1} \otimes C_{2}$ as in eq. (6). Assume that $m=t n_{2}$ is a multiple of $n_{2}$, the length of $C_{2}$. Since $C_{2}$ is selforthogonal, we have that the submatrix of $M$ which consists of the last $m$ columns of $M$ is orthogonal to the submatrix which consists of the first $m$ columns of $M$. Hence, we obtain a semi-infinite stabilizer matrix $S$ by iterative shifting of the block $M$ by $n_{1} n_{2}-m=\left(n_{1}-t\right) n_{2}$ positions.

To give an example, we let $C=[7,3,4]_{2}$ be the Euclidean dual of the binary Hamming code. Using $C \subseteq C^{\perp}=[7,4,3]_{2}$, a QECC $\mathcal{C}=\llbracket 7,1,3 \rrbracket_{2}$ can be constructed. The product code of $C$ with itself is a code $C_{\pi}=C \otimes C=[49,9,16]_{2}$ which is contained in its dual $C_{\pi}^{\perp}=[49,40,3]_{2}$. Hence we obtain a $\mathrm{QECC} \mathcal{C}_{\pi}=\llbracket 49,31,3 \rrbracket_{2}$. The possible parameters for quantum convolutional codes obtained from the product code $C_{\pi}$ by the CSS construction (i.e., by considering the generator matrix $\left.C_{\pi} \otimes G F(4)\right)$ are $(49-m, 31, m), m=7,14$. The free distance of these codes is 3 . Using tail-biting with $N \geq 2$ blocks and $m=7$ (see [8]) we obtain QECCs $\llbracket 42 N, 24 N, d \rrbracket_{2}$. Using Magma [4] we compute $d=3$.

From the product code $\mathcal{C}_{\pi}=\llbracket 15,7,3 \rrbracket_{2}$ described above we can obtain a quantum convolutional code with parameters $(10,7,5)$, i. e., we choose $m=5$.

If the matrix $M$ defining the semi-infinite band matrix $S$ is the generator matrix $G^{(1)} \otimes G^{(2)}$ of a product code, the matrix $S$ itself can be decomposed as a tensor product $S=$ $S^{(1)} \otimes G^{(2)}$, provided the overlap $m$ is a multiple of the length $n_{2}$ of the second code, i. e., $m=t n_{2}$ (see Fig. 4). The matrix 


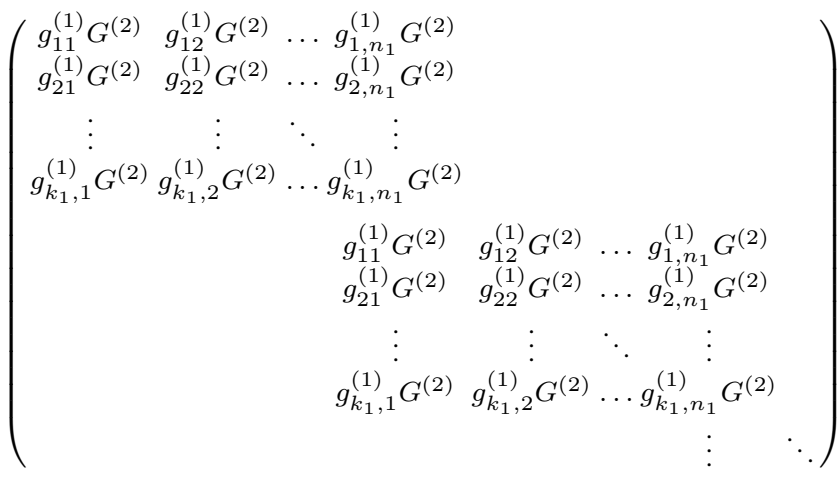

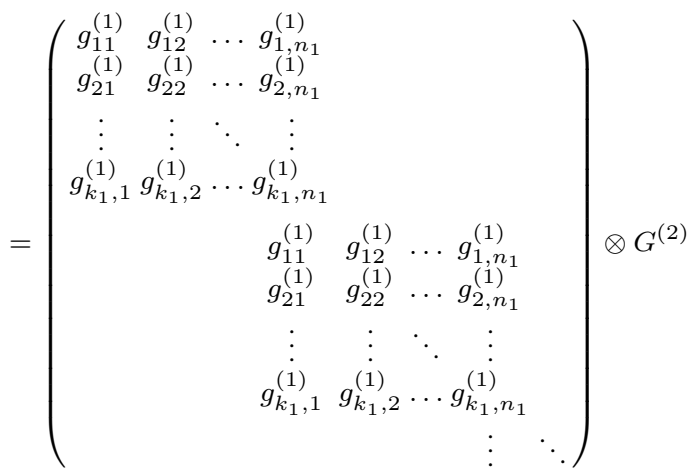

Fig. 4. Tensor product decomposition of the semi-infinite band matrix derived from the generator matrix of a product code (here shown for $t=1$ ).

$S^{(1)}$ is a semi-infinite band matrix with $M^{(1)}=G^{(1)}$ and overlap $t$. From Theorem 7 it follows that the product code is self-orthogonal if $C_{2}$ is self-orthogonal. Hence we get the following construction:

Theorem 10: Let $C_{1}$ be a classical convolutional code. Furthermore, let $C_{2}$ be a self-orthogonal code. Then the product code $C_{1} \otimes C_{2}$ defines a quantum convolutional code, provided at least one of the following holds:

(i) Both $C_{1}$ and $C_{2}$ are linear over $G F(q)$ and $C_{2}$ is Euclidean self-orthogonal.

(ii) Both $C_{1}$ and $C_{2}$ are linear over $G F\left(q^{2}\right)$ and $C_{2}$ is Hermitian self-orthogonal.

(iii) $C_{1}$ is linear of $G F(p)$ and $C_{2}$ is a symplectic selforthogonal code over $G F\left(p^{\ell}\right)$.

\section{CONCLUSION}

The construction of new examples of quantum convolutional codes is a challenging task and rises several questions: what is a general framework to describe such codes, how can they be constructed, and what are the figures of merit to compare the performance of such codes? While the first of these questions has been answered in a satisfying way at least for convolutional stabilizer codes in [13], the other two questions are open (but see e.g. [7], [8], [12]). In this paper we have contributed to the second question by establishing a connection between product codes and convolutional codes. We have shown that the dual distance of product codes can be bounded from below which allows to obtain quantum codes for which the minimum distance is at least as large as the smaller of the minimum distances of the factors.

Concerning the third question currently not much is known, e.g., the significance of notions such as free distance which are useful for classical convolutional codes to the quantum case has yet to be investigated.

\section{ACKNOWLEDGMENT}

This work was carried out while the second author was visiting IAKS. M.R. also acknowledges support by the Institute of Quantum Computing, University of Waterloo. Funding by Deutsche Forschungsgemeinschaft (DFG), Schwerpunktprogramm Quanten-Informationsverarbeitung (SPP 1078), Projekt AQUA (Be 887/13) is acknowledged as well.

\section{REFERENCES}

[1] A. Ashikhmin and E. Knill, "Nonbinary quantum stabilizer codes," IEEE Transactions on Information Theory, vol. 47, no. 7, pp. 3065-3072, Nov. 2001, Preprint quant-ph/0005008

[2] R. E. Blahut, Theory and Practice of Error Control Codes. Reading: Addison-Wesley, 1983.

[3] — Algebraic Codes for Data Transmission. Cambridge: Cambridge University Press, 2003.

[4] W. Bosma, J. J. Cannon, and C. Playoust, "The Magma Algebra System I: The User Language," Journal of Symbolic Computation, vol. 24, no. 3-4, pp. 235-266, 1997.

[5] A. R. Calderbank and P. W. Shor, "Good quantum error-correcting codes exist," Physical Review A, vol. 54, no. 2, pp. 1098-1105, Aug. 1996, preprint quant-ph/9512032

[6] R. T. Chien and S. W. Ng, "Dual Product Codes for Correction of Multiple Low-Density Burst Errors," IEEE Transactions on Information Theory, vol. 19, no. 5, pp. 672-677, Sept. 1973.

[7] A. C. A. de Almeida and R. Palazzo, Jr., "A Concatenated $[(4,1,3)]$ Quantum Convolutional Code," in 2004 IEEE Information Theory Workshop, San Antonio, TX, Oct. 2004.

[8] G. D. Forney, Jr. and S. Guha, "Simple rate- $1 / 3$ convolutional and tail-biting quantum error-correcting codes," in Proc. ISIT'05, Adelaide, Australia, 2005, pp. 1028-1032.

[9] M. Grassl and Th. Beth, "Cyclic quantum error-correcting codes and quantum shift registers," Proceedings of the Royal Society London A, vol. 456, no. 2003, pp. 2689-2706, Nov. 2000, preprint quant-ph/9910061

[10] M. Grassl, Th. Beth, and M. Rötteler, “On Optimal Quantum Codes,' International Journal of Quantum Information, vol. 2, no. 1, pp. 55-64, 2004, preprint quant-ph/0312164

[11] F. J. MacWilliams and N. J. A. Sloane, The Theory of Error-Correcting Codes. Amsterdam: North-Holland, 1977.

[12] H. Ollivier and J.-P. Tillich, "Description of a quantum convolutional code," Physical Review Letters, vol. 91, 177902, Oct. 242003.

[13] —, "Quantum convolutional codes: fundamentals," Nov. 2004 preprint quant-ph/0401134

[14] A. M. Steane, "Error Correcting Codes in Quantum Theory," Physical Review Letters, vol. 77, no. 5, pp. 793-797, 29. July 1996.

[15] J. K. Wolf, "On Codes Derivable from the Tensor Product of Check Matrices," IEEE Transactions on Information Theory, vol. 11, no. 2, pp. 281-284, Apr. 1965. 